\title{
Quality of Life Assessment in Cancer Patients of Regional Centre of Hyderabad City
}

\author{
V. Naga Sunanda ${ }^{1}$, M. Priyanka ${ }^{1}$, J. Architha $^{1}$, M. Shravan ${ }^{1}$, A. Srinivasa Rao ${ }^{2}$, Mohd. Abdul Hadi $^{3}$ \\ ${ }^{1}$ Doctor of Pharmacy, Bhaskar Pharmacy College, Yenkapally (V), Moinabad (M), R. R. District, Hyderabad-500075, Telangana, India. \\ ${ }^{2}$ Bhaskar Pharmacy College, Yenkapally (V), Moinabad (M), R. R. District, Hyderabad-500075, Telangana, India. \\ ${ }^{3}$ Department of Pharmaceutics, Bhaskar Pharmacy College, Yenkapally (V), Moinabad (M), R. R. District, Hyderabad-500075, Telangana, India.
}

\begin{tabular}{|c|c|}
\hline ARTICLE INFO & ABSTRACT \\
\hline Article history: & \multirow{9}{*}{$\begin{array}{l}\text { The present study was carried out to determine the quality of life in regional cancer patients of Hyderabad city } \\
\text { with an objective to create awareness about the various health related issues and financial problems underlying } \\
\text { the disease. So that possible measures can be taken by the society and the government in advance to improve the } \\
\text { quality of life in cancer patients. The complete data for the present study has been collected for a period of } 2 \\
\text { months from Mehdi Nawaz Jung Institute of Oncology and Regional Cancer Centre, Red Hills, Hyderabad, } \\
\text { Telangana, India from } 192 \text { Females and } 32 \text { Males in the age group between } 18-70 \text { years. The quality of life of } \\
\text { the cancer subjects was assessed using EORTC QLQ- C-30 questionnaire. The observations have shown that the } \\
\text { cancer patients in spite of having better functioning and minimum symptoms, their perception was that they had } \\
\text { poor quality of life. It is concluded that the therapy should be individualized for each patient not just based upon } \\
\text { the type or stage of cancer but also based on the patient's priorities, concerns and symptoms along with treating } \\
\text { the disease. In simple words it can be said that the therapy should be patient oriented rather than disease } \\
\text { oriented. }\end{array}$} \\
\hline Received on: 18/10/2017 & \\
\hline Accepted on: $24 / 12 / 2017$ & \\
\hline Available online: $28 / 01 / 2018$ & \\
\hline & \\
\hline & \\
\hline Hyderabad city; EORTC & \\
\hline QLQ- C-30 questionnaire; & \\
\hline chemotherapy. & \\
\hline
\end{tabular}

\section{INTRODUCTION}

Having a potentially life-threatening disease like cancer often makes people to examine their lives and look for meaning. In fact, this search for meaning can be the aspect of cancer that most often has a positive influence on life (Th-iboldeaux and Golant., 2012). The fear of death that affects most people when they are diagnosed with cancer, often makes them to think about what they will leave behind and what they would like to do with the time left. It can make people feel that it's the quality of life (QoL), not just the quantity, which matters the most (Finelli., 2017).

\footnotetext{
* Corresponding Author

V. Naga Sunanda, Doctor of Pharmacy, Bhaskar Pharmacy College, Yenkapally (V), Moinabad (M), R.R District, Hyderabad-500075, Telangana, India.

E-Mail: vema.nagasunanda@gmail.com

Cell no: +919491868106
}

Today in many cultures and societies, cancer remains a taboo and the people suffering with cancer are subjected to stigma and discrimination which prevents them from seeking care (Valerie et al., 2015). The cancer disease can have a severe impact on a person's physical, mental and emotional states and also keep them at more risk of diminished quality of life for several years after diagnosis (Harden et al., 2008). The physiologic effects of some cancer treatments such as hair loss, sexual dysfunction, impaired fertility and weight gain can also leads to stigma and discrimination and sometimes can be the cause of partner rejection (Aubin and Perez, 2015). The psychological toll for caring a cancer living person can also be enormous as many care givers experiences distress and declines in their physical and mental health (Adler and Page, 2008). The pain of cancer experienced due to inadequate access to pain relieving medicines has wide implications in the quality of life of cancer patients and is frequently linked to psychological distress, including higher levels of anxiety, depression and fear (Wells et al., 2008). 
Even though the morphine is considered as an essential medicine by the World Health Organization, there are unacceptable differences in worldwide use of opioids for the pain treatment. Because, $93 \%$ of the world's morphine supply is consumed by high-income countries itself while $65 \%$ of cancer deaths occur in low and middle-income countries (Human Rights Watch., 2009).

The general well-being of individuals and societies is determined by Quality of life which outlines the positive and negative features of life. It includes life satisfaction including everything from physical health, education, family, religious beliefs, employment, wealth, finance and environment. Health care professionals such as physician, nurse and pharmacist observes quality of life from the angle of therapeutic outcome. In case of diseased conditions shorten the life expectancy and for which there is no cure, then maintaining functional well-being and managing symptoms should be the main objective of the health care. There are four possible standards of QoL associated with pharmacotherapy, such as QoL is properly maintained, QoL is improved, QoL is decreased and QoL remains unaffected. These outcomes can be assessed or estimated with the help of physical, biological and other manifestations of the diseases (EORTCQuality of Life; Megari., 2013).

In the present research, we aimed to determine the quality of life in regional cancer patients of Hyderabad city with an objective to create awareness about the various health related issues and financial problems underlying the disease. So that possible measures can be taken by the society and the government in advance to improve the quality of life in cancer patients.

\section{MATERIALS AND METHODS}

The protocol of the present research was approved by the Institutional Ethics Committee, Mehdi Nawaz Jung Institute of Oncology and Regional Cancer Centre, Red Hills, Hyderabad, Telangana, India on $28^{\text {th }}$ November, 2016. The complete data for the present study has been collected for a period of 2 months from 192 Females and 32 Males at the similar above mentioned Regional Cancer Centre. The subjects included in the research were of the age group 18-70 years, who were conscious, cooperative and were undergoing chemotherapy. The present study excluded the subjects who were not able to communicate verbally and had serious psychiatric disorders. Most of the study subjects from the hospital were of Telangana origin, and as this is a government hospital and majority of the study subjects belonged to the lower and middle class families with a monthly salary ranging from Rs.10,000/- to Rs.30,000/- and their complete treatment costs were funded by the government. During the study at the regional centre, it was found that females were more affected with cancer when compared to males.

Majority of the patients had to work hard to support their families but after being affected by cancer there was both physical and mental impairment in their occupations. For the study purpose, initially the data of various parameters such as age, sex, occupation, financial status, cancer type, regimens and comorbidities were collected from the patients and their case sheets which were available bedside in the hospital. The quality of life of the cancer subjects was assessed using European Organization of Research and Treatment in Cancer - Quality of Life Questionnaire C-30 (EORTC QLQ C-30).

It is a questionnaire for patient self-completion, containing multi-items and single scales. It is divided into 5 functional scales (i.e. physical, role, emotional, social and cognitive), 3 symptoms scales (i.e. fatigue, pain, nausea and vomiting) and 6 single items (i.e. dyspnea, insomnia, appetite loss, constipation, diarrhea, financial difficulties) and global health scale. It consists of 30 questions, out of which 2 questions are used to determine global health scale, 15 for functional scale and 13 for symptoms scales. Based on all these scales the overall quality of life of subjects was assessed.

The questionnaire was given to the patients to fill and each question was given score ranging 1 to 4 . In this range, score 1 indicates "Not at all", score 2 indicates "A little", score 3 indicates "quiet a bit" and score 4 indicates "Very much". Based on the patients perspective, scoring was done for all the questions and raw score (RS) which is the mean of component items was calculated for all the scales.

The formula for calculating raw score is:

Raw Score $=$

Sum of scores of questions pertaining to each functioning or symptom Number of questions pertaining to each functioning or symptom

Then, for functional scale: Score $=1-\frac{(R S-1)}{\text { range }} \times 100$

and for Symptom scales/items and global health status:

$$
\text { Score }=\{(R S-1) / \text { range }\} \times 100
$$

Whereas, range is the difference between maximum and minimum score. As such for functional and symptom scales, the range is 3 and for global health status, the range is 6 .

The scores of all the scales and single-item measures ranged from 0 to 100 . The higher response level was indicated based on a high scale score. Thus, a high scale score for a functional scale represents a high/ healthy level of functioning, for the global health status it represents a high QoL, but a high scale score for a symptom scale/ item represents a higher occurrence of symptoms/ problems (EORTC- Quality of Life; Megari., 2013).

\section{RESULTS AND DISCUSSION}

In our current prospective study, interviews were conducted for 224 cancer patients, 192 of whom were females $(85.7 \%)$ and 32 were males $(14.3 \%)$, this was found to be in concordance with the study conducted by Tadele, 2015 who evaluated the QoL of 388 adult cancer patients of which $64.7 \%$ 
patients were female and $35.3 \%$ were male (Tadele., 2015). The patients of this study attending the outpatient department (OPD) of Regional Cancer Centre of Hyderabad were on chemotherapy and their Quality of life was assessed. Demographic criteria such as age and sex of all the cancer patients are represented in Figures 1a and $1 \mathrm{~b}$ respectively.

The mean age of female and male cancer patients was $54 \pm 16$ and $54 \pm 16$ years respectively. The male to female ratio was found to be 1:6 and they were divided into six groups based on their age. The prevalence of Cancer was found to be more in patients with age greater than 40 years $(67 \%)$. Among 224 cancer patients, majority of them were females, as such $85(37.9 \%)$ of them were diagnosed with breast cancer, followed by $40(17.8 \%)$ patients with cervical cancer and $22(9.8 \%)$ patients with ovarian cancer as represented in Figure 1c. Our results were in concordance with the study conducted by Niguse Tadele, 2015 in which most prevalent types of cancer were breast cancer $29.4 \%$ and cervical cancer $26.3 \%$ (Tadele., 2015). There are multiple factors which contribute for the higher prevalence of Breast cancer such as increased exposure to hormones like early menarche, late menopause, use of hormone replacement therapy, work stress, obesity and high pollution level of urban cities (U.S. Department of Health \& Human Services. 2017).

Cancer is an important health issue and the amount of symptoms experienced by a patient influences his Quality of life (Valerie et al., 2015). So, an increasingly important issue in oncology is to evaluate QoL in cancer patients. Moreover, QoL tool is increasingly being used by clinical researchers as a primary outcome measure in studies to evaluate the effectiveness of treatment so that proper symptomatic therapy can be provided to them in order to enhance their Quality of Life. The QoL of individual cancer patients was assessed using EORTC QLQ-C30 questionnaire which contains global health scale, functional scale and symptom scale. Each scale scores were categorized into 4 groups with a range of 25 each (EORTC- Quality of Life; Megari., 2013).

The GHS indicates the entire QoL of the patients, as the score " 0 " and " 100 " indicates very poor and very good quality of life respectively. From the study, it was found that majority of patients i.e. $143(63.8 \%)$ had a GHS score in the range of 26-50 as represented in Figure 2a. Hence, the GHS results indicate that the majority of patients had lower than normal quality of life.

As functional scale includes five different scales such as physical, role, emotional, cognitive and social functioning, the scores of all the individual scales were calculated and the average of all the scores are plotted as AFS. The higher the AFS scores, better the QoL (EORTC- Quality of Life, Megari., 2013). In our study, it was found that 107 patients (47.8\%) and 94 patients (42\%) had an AFS score in the range of 51-75 and 76-100 respectively as represented in Figure $2 b$. Hence, the AFS results indicate that the majority of patients had better functioning. The least affected in majority of the cancer patients was found to be social functioning (mean- 91.22 \pm 1.2 ) and the most affected was found to be emotional functioning (mean-52.16 \pm 2.0 ) as represented in Figure 2c. The Higher ASS score indicates that the patients had more symptoms, and lower the ASS score indicates better the QoL. From the study, it was found that $98(43.8 \%)$ and 93 patients $(41.5 \%)$ had an ASS score in the range of 26-50 and 025 respectively as represented in Figure $2 \mathrm{~d}$. No patients were found to have symptoms in the range of 76-100 which means none of them had extreme symptoms. Hence, the ASS results indicate that the majority of patients had better QoL. A total of nine symptom scales are present to assess the extent of symptoms experienced by the cancer patients. The maximum symptoms experienced by patients were found to be fatigue $(48.6 \pm 1.66)$, followed by pain $(48.06 \pm 1.95)$, sleep $(46.13 \pm 2.82)$ and loss of appetite (45.24 \pm 2.54$)$ as represented in Figure 2e. Our results were in concordance with the study conducted by Kawaguchi et al., 2012 and Charalambous et al., 2016 (Kawaguchi et al., 2012; Charalambous et al., 2016).

During the observational study by using EORTC QLQC30 questionaire, it was found that majority of patients were suffering from various physical and emotional/ psychological problems. In physical problems, pain and cancer related fatigue was experienced by majority of the patients. Whereas, emotionally the patients were more worried about their health, disturbance in their daily activities, occupational and social impairment and relationship with their family.

The financial status was least affected as it was a government funded hospital. But still as the majority of patients were from poor and middle class background they reported that they faced some financial problems due to transportation charges incurred due to frequent hospital visits for chemotherapy and the daily allowance charges for the attendants.

From the above GHS, AFS and ASS scores, it was found that inspite of having better functioning and minimum symptoms, the patient's perception was that they had poor QoL. This was mainly due to the frequent hospital visits for chemotherapy sessions which were followed by adverse effects and impairment of their professional life, as known during interaction with the patients. Therefore, it is necessary that extra measures have to be taken to minimize the adverse effects due to chemotherapy and paid leaves have to be provided for cancer patients along with medical allowances.

Poor patients suffering from cancer should be given special allowances and financial support so that they should not stop the treatment midway due to financial crisis. Moreover, cancer sub-centers has to be started at all districts level in order to reduce traveling time and costs for patients convenience. Hence, the therapy should be individualized for each patient not just based upon the type or stage of cancer but also based on the patient's priorities, concerns and symptoms along with treating the disease.

To achieve such results, clinical pharmacist's has be appointed in order to counsel and educate the patients regarding the importance of therapy and special life style modifications. So that, the symptoms of disease and adverse effects of the drugs can be overcome based on the individual patient condition. 

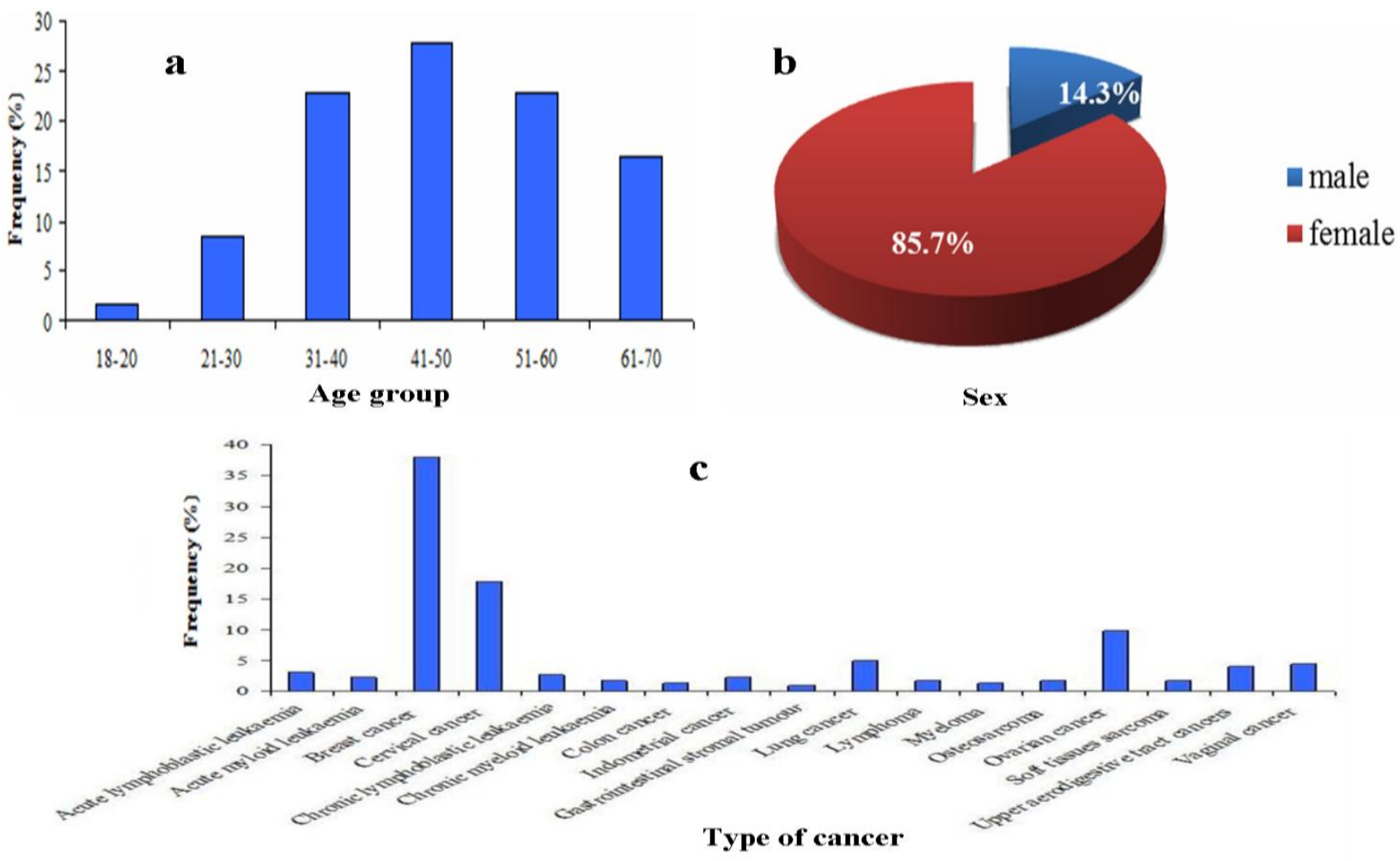

Fig. 1: Data obtained from the regional cancer centre of Hyderabad city such as a) Age b) sex and c) Type of cancer patients.

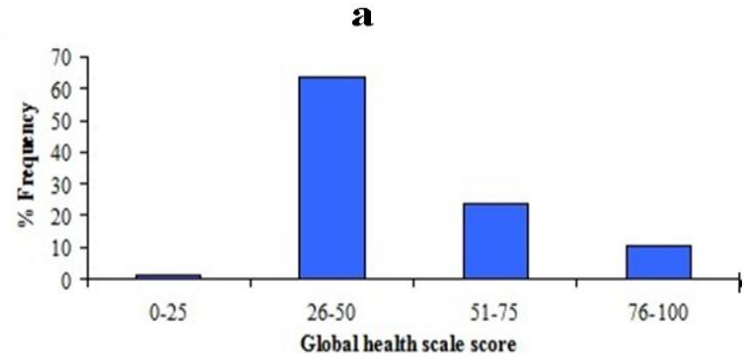

b

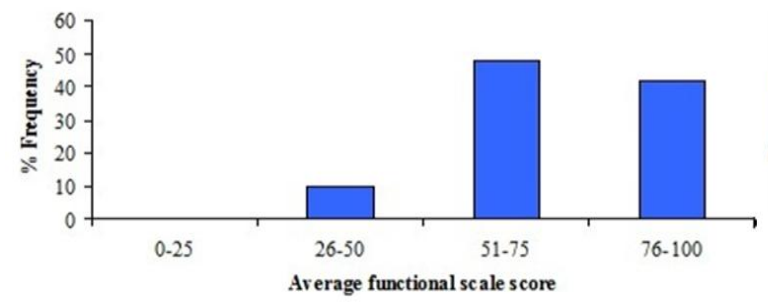

d

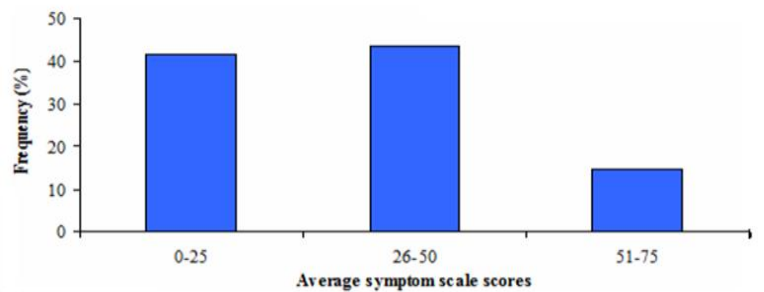

c

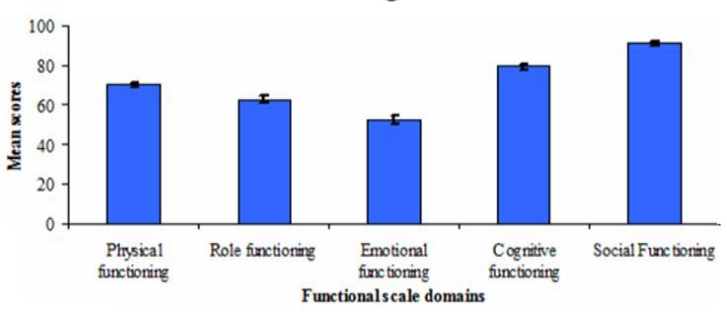

e

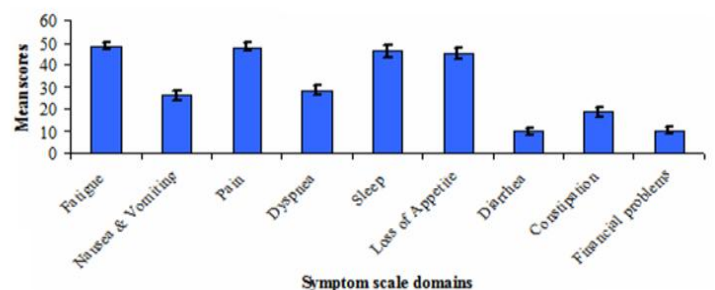

Fig. 2: Data scores of a) Global health scale b) Average functional scale c) Functional scale domains d) Average symptom scale e) Symptom scale domains. 


\section{CONCLUSION}

The quality of life in the regional cancer patients of Hyderabad city was successfully studied using EORTC QLQ C-30 questionnaire with an objective to find out major concerns of the patients suffering from cancer and to create awareness among the health care professionals. From the study, it was observed that the chemotherapy had a significant role in influencing the quality of life. Better functioning and minimum symptoms were observed in majority of patients, but still according to the patients perspective, they had poor quality of life due to frequent hospital visits for chemotherapy sessions which were followed by adverse effects and impairment in their daily and professional activities. Hence, it is concluded that the therapy should be individualized for each patient not just based upon the type or stage of cancer but also based on the patient's priorities, concerns and symptoms along with treating the disease. In simple words it can be said that the therapy should be patient oriented rather than disease oriented. Also, special measures have to be taken to counsel the patients and encouraging them to do activities which they like so that they can be positive and happy in their lives.

\section{ACKNOWLEDGMENT}

The authors are very much thankful to the Chairman of JB group of Educational Institutions Shri. J. V. Krishna Rao Garu for his constant help, support and encouragement to the academics generally and research particularly. The authors are also thankful to Dr. Dayakar, Surgical oncologist, Mehdi Nawaz Jung Institute of Oncology and Regional Cancer Centre, Red Hills, Hyderabad, for the constant help in carrying out the work and providing suitable research facilities.

\section{CONFLICT OF INTEREST}

The authors declare no conflict of interest.

\section{REFERENCES}

Adler NE, Page AEK. 2008. Cancer Care for the Whole Patient: Meeting Psychosocial Health Needs. Washington (DC): National Academies Press (US). Available from: https://www.ncbi.nlm.nih.gov/books/NBK4015/ doi: 10.17226/11993

Andreas Charalambous and Christiana Kouta, "Cancer Related Fatigue and Quality of Life in Patients with Advanced Prostate Cancer Undergoing Chemotherapy," BioMed Res Int, vol. 2016, Article ID 3989286, 11 pages, 2016
Aubin S, Perez S. The Clinician's Toolbox: Assessing the Sexual Impacts of Cancer on Adolescents and Young Adults with Cancer (AYAC). Sex Med. 2015; 3(3): 198-212.

Division of Cancer Prevention and Control, Centers for Disease Control and Prevention. U.S. Department of Health \& Human Services. 2017. Available

at

https://www.cdc.gov/cancer/breast/basic_info/risk_factors.htm

EORTC- Quality of Life. Quality of Life Department, Available at: http://groups.eortc.be/qol/quality-life

Finelli T. Men With Metastatic Prostate Cancer: It's Not Just Quantity Of Life That Matters, But Quality Of Life. Personal health news. Research and Innovations. MediaPlanet 2017. Available at http://www.personalhealthnews.ca/research-and-innovations

Harden J, Northouse L, Cimprich B, Pohl MJ, Liang J, Kershaw $\mathrm{T}$ The Influence of Developmental Life Stage on Quality of Life In Survivors of Prostate Cancer and Their Partners. J Cancer Surviv. 2008; 2(2): 84-94.

Megari K. Quality of Life in Chronic Disease Patients. Health Psychol Res. 2013 Sep 24; 1(3): e27.

Kawaguchi T, Iwase S, Koinuma M, Onodera Y, Takeuchi H, Umeda M, Matsunaga T, Unezaki S, Nagumo Y. Determinants affecting quality of life: implications for pharmacist counseling for patients with breast cancer in Japan. Biol Pharm Bull. 2012; 35(1): 59-64.

Tadele N. Evaluation of quality of life of adult cancer patients attending tikur anbessa specialized referral hospital, Addis ababa Ethiopia. Ethiop J Health Sci. 2015; 25(1): 53-62.

Thiboldeaux K, Golant M. 2012. Reclaiming Your Life After Diagnosis: The Cancer Support Community Handbook. BenBella Books. Page 192.

"Please, do not make us suffer any more..." Access to Pain Treatment as a Human Right. 2009 Human Rights Watch. United States of America. ISBN: 1-56432-449-4, March, 2009.

Valerie F, Wendy L, Paul K. Han, Michael P. Pignone. Decision Making and Cancer. Am Psych. 2015; 70 (2): 105-118.

Wells N, Pasero C, McCaffery M. 2008. Improving the Quality of Care through Pain Assessment and Management. In: Hughes RG, editor. Patient Safety and Quality: An Evidence-Based Handbook for Nurses. Rockville (MD): Agency for Healthcare Research and Quality (US); Chapter $17 . \quad$ Available from: https://www.ncbi.nlm.nih.gov/books/NBK2658/

\section{How to cite this article:}

Sunanda VN, Priyanka M, Architha J, Shravan M, Srinivasa Rao A, Hadi MA. Quality of Life Assessment in Cancer Patients of Regional Centre of Hyderabad City. J App Pharm Sci, 2018; 8 (01): 165-169. 